\title{
THE SCATTERING OF THE SOUND FIELD BY THIN UNCLOSED SPHERICAL SHELL AND ELLIPSOID
}

\begin{abstract}
In this paper the result of solution of the axisymmetric problem of the scattering of sound field by unclosed spherical shell and a soft prolate ellipsoid of rotation is presented. Spherical radiator is located in a thin unclosed spherical shell as the source of acoustic field. The equation of the spheroidal boundary is given in spherical coordinates. Scattered pressure field is expressed in terms of spherical wave functions. Using corresponding theorems of addition and assuming small eccentricity of ellipse, the solution of boundary value problem is reduced to solving dual equations with Legendre's polynomials, which are converted to infinite system of linear algebraic equations of the second kind with completely continuous operator. Numerical results are given for various values of the parameters of the problem.
\end{abstract}

Keywords: sound field, spherical shell, ellipsoid of rotation, spherical radiator

\section{Introduction}

Many researchers have solved the problem of sound scattering on spheroid by different methods. For example, the scattering of the sound field by hard or soft, prolate or oblate spheroids are considered in [1-7]. The results of the scattering of sound permeable and elastic spheroids are studied in the works [8-12]. Analytical description of the acoustic field scattered by inhomogeneous elastic spheroid is obtained in [13]. In [14] analytical solution of the problem of diffractions of plane sound wave on elastic spheroid with arbitrary located spherical cavity is considered.

In this paper analytical solution of the axisymmetric problem of scattering of sound field by unclosed spherical shell and soft prolate ellipsoid of rotation is

\footnotetext{
1 Autor do korespondencji/corresponding author: Gennady Shushkevich, Yanka Kupala State University of Grodno, 22,Ozheshko St., 230023 Grodno, Belarus, e-mail: g_shu@tut.by

${ }^{2}$ Svetlana Shushkevich, Yanka Kupala State University of Grodno, e-mail: spusha@list.ru

${ }^{3}$ Feliks Stachowicz, Rzeszow University of Technology, e-mail: stafel@prz.edu.pl
} 
presented. A spherical radiator was located in the thin unclosed spherical shell as the source of the acoustic field. The equation of spheroidal boundary is given in spherical coordinates. The solution of boundary value problem is reduced to solving dual equations with Legendre's polynomials which are converted to infinite system of linear algebraic equations of the second kind with completely continuous operator. Numerical results are given for various values of parameters of the problem.

\section{Problem formulation}

Let homogeneous space $\mathrm{R}^{3}$ contain a thin unclosed spherical shell $\Gamma_{1}$ located on the sphere $\Gamma$ of radius with the center at the point $\mathrm{O}$ and a prolate ellipsoid of revolution $\mathrm{S}$ where $\mathrm{a}$ is semi-major axis of the ellipse $\mathrm{b}$ is a minor axis of the ellipse $a>b$ (fig. 1). We denote by $D_{1}$ the area of space bounded by the sphere $\Gamma$ and by $\mathrm{D}_{3}$ the area of space bounded by the ellipsoid $\mathrm{S}$. The distance between points $\mathrm{O}$ and $\mathrm{O}_{1}$ is equal to $\mathrm{h}_{1}$. Then $\mathrm{D}_{2}=\mathrm{R}^{3} \backslash\left(\mathrm{D}_{1} \cup \Gamma \cup \mathrm{D}_{3} \cup S\right)$.

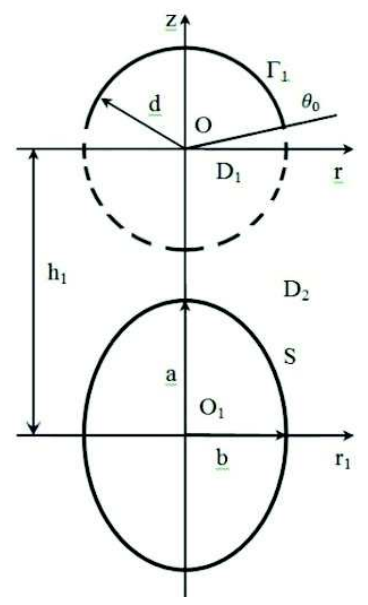

Fig. 1. Geometry of the problem

A point radiator of sound waves oscillating with an angular frequency $\omega$ is located at the point $O$. The areas $D_{j}=1,2$ are filled with the material in which shear waves do not distribute. Let denote the density of medium by $\rho$ and speed of sound by $\mathrm{c}$ in $\mathrm{D}_{\mathrm{j}}$. To solve this problem we connect spherical coordinates with point $\mathrm{O}$ and point $\mathrm{O}_{1}$. Spherical shell $\Gamma_{1}$ and ellipsoidal shell $\mathrm{S}$ are described as follows:

$$
\begin{aligned}
& \Gamma_{1}=\left\{\mathrm{r}=\mathrm{d}, 0 \leq \theta \leq \theta_{0}<\pi, 0 \leq \varphi \leq 2 \pi\right\} \\
& \mathrm{S}=\left\{\mathrm{r}_{1}=\gamma\left(\theta_{1}\right), 0 \leq \theta_{1} \leq \pi, 0 \leq \varphi \leq 2 \pi\right\}
\end{aligned}
$$


where: $\gamma\left(\theta_{1}\right)=\mathrm{a} / \sqrt{1-\mathrm{V} \sin ^{2} \theta_{1}}, \mathrm{~V}=1-(\mathrm{a} / \mathrm{b})^{2}$.

Let $\mathrm{p}_{\mathrm{c}}$ be the pressure of the sound field of the primary point radiator, $\mathrm{p}_{\mathrm{j}}$ is secondary sound pressure field in the area $D_{j}, j=1,2$. The actual sound pressure is calculated by the formula $P_{j}=\operatorname{Re}\left(p_{j} e^{-i \omega t}\right)$. The solution of the diffraction problem is reduced to finding pressures $\mathrm{p}_{\mathrm{j}}, \mathrm{j}=1,2$, which satisfy:

- Helmholtz equation $[15,16]$

$$
\Delta \mathrm{p}_{\mathrm{j}}+\mathrm{k}^{2} \mathrm{p}_{\mathrm{j}}=0
$$

where $\Delta=\frac{\partial^{2}}{\partial \mathrm{x}^{2}}+\frac{\partial^{2}}{\partial \mathrm{y}^{2}}+\frac{\partial^{2}}{\partial \mathrm{z}^{2}}$ is Laplace's operator, $\mathrm{k}=\omega / \mathrm{c}$ is the wave number,

- boundary condition on the surface of spherical shell $\Gamma_{1}$ (acoustically hard shell):

$$
\left.\frac{\mathrm{a}}{\partial \overrightarrow{\mathbf{n}}}\left(\mathrm{p}_{\mathrm{c}}+\mathrm{p}_{1}\right)\right|_{\Gamma_{1}}=0
$$

where $\overrightarrow{\mathbf{n}}$ is the normal to the surface $\Gamma_{1}$,

- boundary conditions on the surface of ellipsoidal shell S (acoustically soft shell):

$$
\left.\mathrm{p}_{2}\right|_{\mathrm{S}}=0
$$

and the condition at infinity [16]:

$$
\lim _{M \rightarrow \infty} r\left(\frac{\partial p_{2}(M)}{\partial r}-i k p_{2}(M)\right)=0
$$

where $\mathrm{M}$ is an arbitrary point at the space.

Condition of continuity of the pressure on the open part of the spherical shell $\Gamma \backslash \Gamma_{1}$ is given by:

$$
\left.\left(\mathrm{p}_{\mathrm{c}}+\mathrm{p}_{1}\right)\right|_{\Gamma \backslash \Gamma_{1}}=\left.\mathrm{p}_{2}\right|_{\Gamma \backslash \Gamma_{1}}
$$

and normal derivative on the surface of the sphere $\Gamma$ is:

$$
\left.\frac{\partial}{\partial \mathrm{r}}\left(\mathrm{p}_{\mathrm{c}}+\mathrm{p}_{1}\right)\right|_{\Gamma}=\left.\frac{\partial}{\partial \mathrm{r}} \mathrm{p}_{2}\right|_{\Gamma}
$$


Initial pressure of the sound field can be represented in the form [16]:

$$
\mathrm{p}_{\mathrm{c}}(\mathrm{r}, \theta)=\mathrm{P} \exp (\mathrm{ikr}) / \mathrm{r}=\mathrm{P} \sum_{\mathrm{n}=0}^{\infty} \mathrm{f}_{\mathrm{n}} \mathrm{h}_{\mathrm{n}}^{(1)}(\mathrm{kr}) \mathrm{P}_{\mathrm{n}}(\cos \theta), \mathrm{f}_{\mathrm{n}}=\mathrm{ik} \delta_{0 \mathrm{n}}
$$

where $\mathrm{h}_{\mathrm{n}}^{(1)}(\mathrm{x})$ are spherical Hankel's functions, $\mathrm{P}_{\mathrm{n}}(\cos \theta)$ are Legendre's polynomials [17], $\delta_{0 \mathrm{n}}$ is Kronecker's delta, $\mathrm{P}$ is a constant.

The pressure of the scattered sound field is represented as superposition of basic solutions of Helmholtz equation in spherical coordinates [18, 19] taking into account the condition at infinity (6):

$$
\begin{aligned}
& \mathrm{p}_{1}(\mathrm{r}, \theta)=\mathrm{P} \sum_{\mathrm{n}=0}^{\infty} \mathrm{c}_{\mathrm{n}} \mathrm{j}_{\mathrm{n}}(\mathrm{kr}) \mathrm{P}_{\mathrm{n}}(\cos \theta), \mathrm{r}<\mathrm{d}, \\
& \mathrm{p}_{2}=\mathrm{p}_{2}^{(1)}(\mathrm{r}, \theta)+\mathrm{p}_{2}^{(2)}\left(\mathrm{r}_{1}, \theta_{1}\right), \\
& \mathrm{p}_{2}^{(1)}(\mathrm{r}, \theta)=\mathrm{P} \sum_{\mathrm{n}=0}^{\infty} \mathrm{x}_{\mathrm{n}} \mathrm{h}_{\mathrm{n}}^{(1)}(\mathrm{kr}) \mathrm{P}_{\mathrm{n}}(\cos \theta), \quad \mathrm{r}>\mathrm{d}, \\
& \mathrm{p}_{2}^{(2)}\left(\mathrm{r}_{1}, \theta_{1}\right)=\mathrm{P} \sum_{\mathrm{n}=0}^{\infty} \mathrm{y}_{\mathrm{n}} \mathrm{h}_{\mathrm{n}}^{(1)}\left(\mathrm{kr}_{1}\right) \mathrm{P}_{\mathrm{n}}\left(\cos \theta_{1}\right), \quad \mathrm{r}_{1}>\gamma\left(\theta_{1}\right),
\end{aligned}
$$

where $\mathrm{j}_{\mathrm{n}}(\mathrm{x})$ are spherical Bessel's functions of first kind [17]. Unknown coefficients $\mathrm{c}_{\mathrm{n}}, \mathrm{x}_{\mathrm{n}}, \mathrm{y}_{\mathrm{n}}$ must be determined from the boundary conditions.

\section{Boundary conditions}

Let's perform boundary conditions (4), (7), (8). For this purpose the function $\mathrm{p}_{2}^{(2)}\left(\mathrm{r}_{1}, \theta_{1}\right)$ through spherical wave functions in the coordinate system with origin at the point $\mathrm{O}$ can be determined using the formula connecting spherical wave functions $[18,19]$ :

$$
\mathrm{h}_{\mathrm{n}}^{(1)}\left(\mathrm{kr}_{1}\right) \mathrm{P}_{\mathrm{n}}\left(\cos \theta_{1}\right)=\sum_{\mathrm{k}=0}^{\infty} \mathrm{A}_{\mathrm{nk}}\left(\mathrm{h}_{1}\right) \mathrm{j}_{\mathrm{k}}(\mathrm{kr}) \mathrm{P}_{\mathrm{k}}(\cos \theta), \quad \mathrm{r}<\mathrm{h}_{1}
$$

Then

$$
\mathrm{p}_{2}^{(2)}(\mathrm{r}, \theta)=\mathrm{P} \sum_{\mathrm{n}=0}^{\infty} \mathrm{p}_{\mathrm{n}} \mathrm{j}_{\mathrm{n}}(\mathrm{kr}) \mathrm{P}_{\mathrm{n}}(\cos \theta), \mathrm{p}_{\mathrm{n}}=\sum_{\mathrm{k}=0}^{\infty} \mathrm{y}_{\mathrm{k}} \mathrm{A}_{\mathrm{kn}}\left(\mathrm{h}_{1}\right)
$$


where

$$
\mathrm{A}_{\mathrm{nk}}\left(\mathrm{h}_{1}\right)=(2 \mathrm{k}+1) \sum_{\sigma=|\mathrm{k}-\mathrm{n}|}^{\mathrm{k}+\mathrm{n}} i^{\sigma+\mathrm{k}-\mathrm{n}} \mathrm{b}_{\sigma}^{(\mathrm{n} 0 \mathrm{k} 0)} \mathrm{h}_{\sigma}^{(1)}\left(\mathrm{kh}_{1}\right),
$$

$\mathrm{b}_{\sigma}^{(\mathrm{n} 0 \mathrm{q} 0)}=(\mathrm{nq} 00 \mid \sigma 0)^{2},(\mathrm{nq} 00 \mid \sigma 0)$ is the Klepshev-Gordona coefficient [16].

According to representations (10)-(12), (15), the boundary condition (5) taking into account the condition of orthogonality of Legendre polynomials on the interval $[0 ; \pi]$ becomes:

$$
\left.\begin{array}{l}
f_{n} \frac{d}{d \xi} h_{n}^{(1)}\left(\xi_{0}\right)+c_{n} \frac{d}{d \xi} j_{n}\left(\xi_{0}\right)=x_{n} \frac{d}{d \xi} h_{n}^{(1)}\left(\xi_{0}\right)+p_{n} \frac{d}{d \xi} j_{n}\left(\xi_{0}\right), \\
\xi_{0}=k d, n=0,1, \ldots
\end{array}\right\}
$$

Let us perform the boundary condition (4) on the surface of the spherical shell and the condition of continuity (7). Let us exclude factors $c_{n}$ in the resulting equations using the representation (17), and we obtain dual equations in Legendre's polynomial:

$$
\left.\begin{array}{l}
\sum_{n=0}^{\infty} x_{n} \frac{d}{d \xi_{0}} h_{n}^{(1)}\left(\xi_{0}\right) P_{n}(\cos \theta)=-\sum_{n=0}^{\infty} p_{n} \frac{d}{d \xi_{0}} j_{n}\left(\xi_{0}\right) P_{n}(\cos \theta), \quad 0 \leq \theta<\theta_{0}, \\
\sum_{n=0}^{\infty} \frac{x_{n}-f_{n}}{\frac{d}{d \xi_{0}} j_{n}\left(\xi_{0}\right)} P_{n}(\cos \theta)=0, \quad \theta_{0}<\theta \leq \pi .
\end{array}\right\}
$$

Let new coefficients be

$$
x_{n}=X_{n} \frac{d}{d \xi_{0}} j_{n}\left(\xi_{0}\right)+f_{n}, n=0,1, \ldots
$$

and a small parameter is

$$
g_{n}=1+\frac{4 i \xi_{0}^{3}}{2 n+1} \frac{d}{d \xi_{0}} j_{n}\left(\xi_{0}\right) \frac{d}{d \xi_{0}} h_{n}^{(1)}\left(\xi_{0}\right), g_{n}=O\left(n^{-2}\right), n \gg \xi_{0}
$$

As a result dual equations (18) take the form: 


$$
\left.\begin{array}{l}
\left.\sum_{n=0}^{\infty}(2 n+1)\left(1-g_{n}\right) X_{n} P_{n}(\cos \theta)=\sum_{n=0}^{\infty}(2 n+1)\left(\tilde{f}_{n}+\tilde{p}_{n}\right) P_{n} \mid \cos \theta\right), 0 \leq \theta<\theta_{0}, \\
\sum_{n=0}^{\infty} X_{n} P_{n}(\cos \theta)=0, \quad \theta_{0}<\theta \leq \pi,
\end{array}\right\}
$$

where

$$
\tilde{\mathrm{f}}_{\mathrm{n}}=4 \mathrm{i} \xi_{0}^{3} \mathrm{f}_{\mathrm{n}} \frac{\mathrm{d}}{\mathrm{d} \xi_{0}} \mathrm{~h}_{\mathrm{n}}^{(1)}\left(\xi_{0}\right) /(2 \mathrm{n}+1), \quad \tilde{\mathrm{p}}_{\mathrm{n}}=4 \mathrm{i} \xi_{0} \mathrm{p}_{\mathrm{n}} \frac{\mathrm{d}}{\mathrm{d} \xi_{0}} \mathrm{j}_{\mathrm{n}}\left(\xi_{0}\right) /(2 \mathrm{n}+1)
$$

Dual equations (18) are converted to infinite system of linear algebraic equations of the second kind with the completely continuous operator using the integral representation for Legendre's polynomials $[19,20]$ :

$$
\mathrm{X}_{\mathrm{n}}-\sum_{\mathrm{k}=0}^{\infty} \mathrm{g}_{\mathrm{k}} \mathrm{R}_{\mathrm{nk}}\left(\theta_{0}\right) \mathrm{X}_{\mathrm{k}}=\sum_{\mathrm{k}=0}^{\infty}\left(\tilde{\mathrm{p}}_{\mathrm{k}}+\tilde{\mathrm{f}}_{\mathrm{k}} \mid \mathrm{R}_{\mathrm{nk}}\left(\theta_{0}\right), \quad \mathrm{n}=0,1, \ldots,\right.
$$

where

$$
\left.\begin{array}{l}
\mathrm{R}_{\mathrm{nk}}\left(\theta_{0}\right)=\frac{1}{\pi}\left[\frac{\sin (\mathrm{n}-\mathrm{k}) \theta_{0}}{\mathrm{n}-\mathrm{k}}-\frac{\sin (\mathrm{n}+\mathrm{k}+1) \theta_{0}}{\mathrm{n}+\mathrm{k}+1}\right], \\
\left.\frac{\sin (\mathrm{n}-\mathrm{k}) \theta_{0}}{\mathrm{n}-\mathrm{k}}\right|_{\mathrm{n}=\mathrm{k}}=\theta_{0} .
\end{array}\right\},
$$

To analyze boundary conditions (5) we express the function $\mathrm{p}_{2}^{(1)}(\mathrm{r}, \theta)$ through spherical wave functions in the coordinate system with origin at the point $\mathrm{O}$ using formula $[18,19]$ :

$$
\mathrm{h}_{\mathrm{n}}^{(1)}(\mathrm{kr}) \mathrm{P}_{\mathrm{n}}(\cos \theta)=\sum_{\mathrm{k}=0}^{\infty} \mathrm{B}_{\mathrm{nk}}\left(\mathrm{h}_{1}\right) \mathrm{j}_{\mathrm{k}}\left(\mathrm{kr}_{1}\right) \mathrm{P}_{\mathrm{k}}\left(\cos \theta_{1}\right), \mathrm{r}_{1}<\mathrm{h}_{1}
$$

then

$$
\mathrm{p}_{2}^{(1)}\left(\mathrm{r}_{1}, \theta_{1}\right)=\mathrm{P} \sum_{\mathrm{n}=0}^{\infty} \mathrm{z}_{\mathrm{n}} \mathrm{j}_{\mathrm{n}}\left(\mathrm{kr}_{1}\right) \mathrm{P}_{\mathrm{n}}\left(\cos \theta_{1}\right), \mathrm{z}_{\mathrm{n}}=\sum_{\mathrm{p}=0}^{\infty} \mathrm{x}_{\mathrm{p}} \mathrm{B}_{\mathrm{pn}}\left(\mathrm{h}_{1}\right)
$$

where 


$$
\mathrm{B}_{\mathrm{nk}}\left(\mathrm{h}_{1}\right)=(2 \mathrm{k}+1) \sum_{\sigma=|\mathrm{k}-\mathrm{n}|}^{\mathrm{k}+\mathrm{n}}(-1)^{\sigma} \mathrm{i}^{\sigma+\mathrm{k}-\mathrm{n}} \mathrm{b}_{\sigma}^{(\mathrm{n} 0 \mathrm{k} 0)} \mathrm{h}_{\sigma}^{(1)}\left(\mathrm{kh} \mathrm{h}_{1}\right)
$$

Taking into account the representation (13), (26) and boundary conditions (5) we obtain

$$
\sum_{n=0}^{\infty} \mathrm{z}_{\mathrm{n}} \mathrm{j}_{\mathrm{n}}\left(\mathrm{k} \gamma\left(\theta_{1}\right)\right) \mathrm{P}_{\mathrm{n}}\left(\cos \theta_{1}\right)+\sum_{\mathrm{n}=0}^{\infty} \mathrm{y}_{\mathrm{n}} \mathrm{h}_{\mathrm{n}}^{(1)}\left(\mathrm{k}_{0} \gamma\left(\theta_{1}\right)\right) \mathrm{P}_{\mathrm{n}}\left(\cos \theta_{1}\right)=0
$$

We transform the relation (28) and assume that the eccentricity of ellipse is $\mathrm{h}=\sqrt{1-\mathrm{b}^{2} / \mathrm{a}^{2}}<<1, \mathrm{a}>\mathrm{b}$, then

$$
\left.\begin{array}{l}
\mathrm{V}=-\mathrm{h}^{2}-\mathrm{h}^{4}-\mathrm{h}^{6}+\mathrm{O}\left(\mathrm{h}^{8}\right), \quad \gamma\left(\theta_{1}\right)=\mathrm{a}\left[1-\frac{\mathrm{h}^{2}}{2} \sin ^{2} \theta_{1}-\frac{\mathrm{h}^{4}}{2}\left(\sin ^{2} \theta_{1}-\right.\right. \\
\left.\left.-\frac{3}{4} \sin ^{4} \theta_{1}\right)-\frac{\mathrm{h}^{6}}{2}\left(\sin ^{2} \theta_{1}-\frac{3}{2} \sin ^{4} \theta_{1}+\frac{5}{8} \sin ^{6} \theta_{1}\right)\right]+\mathrm{O}\left(\mathrm{h}^{8}\right) .
\end{array}\right\}
$$

Now we factorize spherical functions $\mathrm{j}_{\mathrm{n}}\left(\gamma\left(\theta_{1}\right)\right), \mathrm{h}_{\mathrm{n}}^{(1)}\left(\gamma\left(\theta_{1}\right)\right)$ in series with respect to small parameter h:

$$
\left.\begin{array}{l}
\mathrm{j}_{\mathrm{n}}\left(\mathrm{k} \gamma\left(\theta_{1}\right)\right)=\mathrm{j}_{\mathrm{n}}\left(\xi_{1}\right)-\frac{\sin ^{2} \theta_{1}}{2} \xi_{1} \mathrm{j}_{\mathrm{n}}^{\prime}\left(\xi_{1}\right) \mathrm{h}^{2}-\left(\xi_{1} \mathrm{j}_{\mathrm{n}}^{\prime}\left(\xi_{1}\right)\left(\frac{\sin ^{2} \theta_{1}}{2}-\frac{3 \sin ^{4} \theta_{1}}{8}\right)-\right. \\
\left.-\frac{\xi_{1}^{2} \mathrm{j}_{\mathrm{n}}^{\prime \prime}\left(\xi_{1}\right)}{8} \sin ^{4} \theta_{1}\right) \mathrm{h}^{4}-\left(\xi_{1} \mathrm{j}_{\mathrm{n}}^{\prime}\left(\xi_{1}\right)\left(\frac{5 \sin ^{6} \theta_{1}}{16}-\frac{3 \sin ^{4} \theta_{1}}{4}+\frac{\sin ^{2} \theta_{1}}{2}\right)-\right. \\
\left.-\xi_{1}^{2} \mathrm{j}_{\mathrm{n}}^{\prime \prime}\left(\xi_{1}\right)\left(\frac{\sin ^{4} \theta_{1}}{4}-\frac{3 \sin ^{6} \theta_{1}}{16}\right)+\frac{\xi_{1}^{3} \mathrm{j}_{\mathrm{n}}^{\prime \prime \prime}\left(\xi_{1}\right) \sin ^{6} \theta_{1}}{48}\right) \mathrm{h}^{6}+\mathrm{O}\left(\mathrm{h}^{8}\right), \xi_{1}=\mathrm{ka} .
\end{array}\right\}
$$

Similar expansion as (30) holds for the function $\mathrm{h}_{\mathrm{n}}^{(1)}\left(\gamma\left(\theta_{1}\right)\right)$, but instead of the function $\mathrm{j}_{\mathrm{n}}\left(\xi_{1}\right)$ is the function $\mathrm{h}_{\mathrm{n}}^{(1)}\left(\xi_{1}\right)$.Expansions for spherical functions can be written as follows:

$$
\left.\begin{array}{l}
\mathrm{j}_{\mathrm{n}}\left(\mathrm{k} \gamma\left(\theta_{1}\right)\right)=\mathrm{p}_{\mathrm{n}}^{(0)}\left(\xi_{1}\right)+\mathrm{p}_{\mathrm{n}}^{(1)}\left(\xi_{1}\right) \sin ^{2} \theta_{1}+\mathrm{p}_{\mathrm{n}}^{(2)}\left(\xi_{1}\right) \sin ^{4} \theta_{1}+\mathrm{p}_{\mathrm{n}}^{(3)}\left(\xi_{1}\right) \sin ^{6} \theta_{1} \\
\mathrm{~h}_{\mathrm{n}}^{(1)}\left(\mathrm{k} \gamma\left(\theta_{1}\right)\right)=\mathrm{m}_{\mathrm{n}}^{(0)}\left(\xi_{1}\right)+\mathrm{m}_{\mathrm{n}}^{(1)}\left(\xi_{1}\right) \sin ^{2} \theta_{1}+\mathrm{m}_{\mathrm{n}}^{(2)}\left(\xi_{1}\right) \sin ^{4} \theta_{1}+\mathrm{m}_{\mathrm{n}}^{(3)}\left(\xi_{1}\right) \sin ^{6} \theta_{1}
\end{array}\right\}
$$


where

$$
\left.\begin{array}{l}
\mathrm{p}_{\mathrm{n}}^{(0)}\left(\xi_{1}\right)=\mathrm{j}_{\mathrm{n}}\left(\xi_{1}\right), \quad \mathrm{p}_{\mathrm{n}}^{(1)}\left(\xi_{1}\right)=-\xi_{1}\left(\mathrm{~h}^{2}+\mathrm{h}^{4}+\mathrm{h}^{6}\right) \mathrm{j}_{\mathrm{n}}^{\prime}\left(\xi_{1}\right) / 2, \\
\mathrm{p}_{\mathrm{n}}^{(2)}\left(\xi_{1}\right)=\left(3 \mathrm{~h}^{4}+6 \mathrm{~h}^{6}\right) \xi_{1} \mathrm{j}_{\mathrm{n}}^{\prime}\left(\xi_{1}\right) / 8+\left(\mathrm{h}^{4}+2 \mathrm{~h}^{6}\right) \xi_{1}^{2} \mathrm{j}_{\mathrm{n}}^{\prime \prime}\left(\xi_{1}\right) / 8, \\
\mathrm{p}_{\mathrm{n}}^{(3)}\left(\xi_{1}\right)=-\left(15 \xi_{\mathrm{n}}^{\prime}\left(\xi_{1}\right)+9 \xi_{1}^{2} \mathrm{j}_{\mathrm{n}}^{\prime \prime}\left(\xi_{1}\right)+\xi_{1}^{3} \mathrm{j}_{\mathrm{n}}^{\prime \prime \prime}\left(\xi_{1}\right)\right) \mathrm{h}^{6} / 48, \\
\mathrm{~m}_{\mathrm{n}}^{(0)}\left(\xi_{1}\right)=\mathrm{h}_{\mathrm{n}}^{(1)}\left(\xi_{1}\right),{ }_{\mathrm{n}}^{(1)}\left(\xi_{1}\right)=-\xi_{1}\left[\mathrm{~h}^{2}+\mathrm{h}^{4}+\mathrm{h}^{6}\right]\left(\mathrm{h}_{\mathrm{n}}^{(1)}\left(\xi_{1}\right)\right)^{\prime} / 2, \\
\mathrm{~m}_{\mathrm{n}}^{(2)}\left(\xi_{1}\right)=\left(3 \mathrm{~h}^{4}+6 \mathrm{~h}^{6}\right) \xi_{1}\left(\mathrm{~h}_{\mathrm{n}}^{(1)}\left(\xi_{1}\right)\right)^{\prime} / 8+\left(\mathrm{h}^{4}+2 \mathrm{~h}^{6}\right) \xi_{1}^{2}\left(\mathrm{~h}_{\mathrm{n}}^{(1)}\left(\xi_{1}\right)\right)^{\prime \prime} / 8, \\
\mathrm{~m}_{\mathrm{n}}^{(3)}\left(\xi_{1}\right)=-\left[15 \xi_{1}\left(\mathrm{~h}_{\mathrm{n}}^{(1)}\left(\xi_{1}\right)\right)^{\prime}+9 \xi_{1}^{2}\left(\mathrm{~h}_{\mathrm{n}}^{(1)}\left(\xi_{1}\right)\right)^{\prime \prime}+\xi_{1}^{3}\left(\mathrm{~h}_{\mathrm{n}}^{(1)}\left(\xi_{1}\right)\right)^{\prime \prime \prime}\right] \mathrm{h}^{6} / 48 .
\end{array}\right\}
$$

Let us exclude factors $z_{n}$ in (28) using the representations (27), (19) and expansions (31). We multiply the resulting equation by $\mathrm{P}_{\mathrm{s}}(\cos \theta) \sin \theta \mathrm{d} \theta, \mathrm{s}=0,1$, $2, \ldots$, and integrate from 0 to $\pi$, then we have:

$$
\left.\sum_{\mathrm{n}=0}^{\infty} \mathrm{X}_{\mathrm{n}} \tilde{\mathrm{a}}_{\mathrm{ns}} \xi_{0}, \xi_{1}, \mathrm{~h}_{1}\right)+\sum_{\mathrm{n}=0}^{\infty} \mathrm{y}_{\mathrm{n}} \mathrm{b}_{\mathrm{ns}}\left(\xi_{1}\right)=-\mathrm{ik} \sum_{\mathrm{n}=0}^{\infty} \mathrm{B}_{0 \mathrm{n}}\left(\mathrm{h}_{1}\right) \mathrm{a}_{\mathrm{ns}}\left(\xi_{1}\right), \mathrm{s}=0,1, \ldots
$$

where

$$
\left.\begin{array}{l}
\left.\tilde{a}_{\mathrm{ns}} \| \xi_{0}, \xi_{1}, \mathrm{~h}_{1}\right)=\frac{\mathrm{d}}{\mathrm{d} \xi_{0}} \mathrm{j}_{\mathrm{n}}\left(\xi_{0}\right) \sum_{\mathrm{m}=0}^{\infty} \mathrm{B}_{\mathrm{nm}}\left(\mathrm{h}_{1}\right) \mathrm{a}_{\mathrm{ms}}\left(\xi_{1}\right), \\
\mathrm{a}_{\mathrm{ns}}\left(\xi_{1}\right)=\mathrm{p}_{\mathrm{n}}^{(0)}\left(\xi_{1}\right) \mathrm{I}_{\mathrm{ns}}^{(1)}+\mathrm{p}_{\mathrm{n}}^{(1)}\left(\xi_{1}\right) \mathrm{I}_{\mathrm{ns}}^{(3)}+\mathrm{p}_{\mathrm{n}}^{(2)}\left(\xi_{1}\right) \mathrm{I}_{\mathrm{ns}}^{(5)}+\mathrm{p}_{\mathrm{n}}^{(3)}\left(\xi_{1}\right) \mathrm{I}_{\mathrm{ns}}^{(7)}, \\
\mathrm{b}_{\mathrm{ns}}\left(\xi_{1}\right)=\mathrm{m}_{\mathrm{n}}^{(0)}\left(\xi_{1}\right) \mathrm{I}_{\mathrm{ns}}^{(1)}+\mathrm{m}_{\mathrm{n}}^{(1)}\left(\xi_{1}\right) \mathrm{I}_{\mathrm{ns}}^{(3)}+\mathrm{m}_{\mathrm{n}}^{(2)}\left(\xi_{1}\right) \mathrm{I}_{\mathrm{ns}}^{(5)}+\mathrm{m}_{\mathrm{n}}^{(3)}\left(\xi_{1}\right) \mathrm{I}_{\mathrm{ns}}^{(7)},
\end{array}\right\}
$$

The values of the integrals $\mathrm{I}_{\mathrm{ns}}^{(\alpha)}$ are given in Appendix. So we have the following connected system of linear algebraic equations for the unknown coefficients from Eqs. (23), (33): 


$$
\left.\begin{array}{l}
\sum_{\mathrm{n}=0}^{\infty}\left(\mathrm{g}_{\mathrm{n}} \mathrm{R}_{\mathrm{sn}}\left(\theta_{0}\right)-\delta_{\mathrm{ns}}\right) \mathrm{X}_{\mathrm{n}}+\sum_{\mathrm{n}=0}^{\infty} \tilde{\mathrm{b}}_{\mathrm{ns}}\left(\xi_{0}, \theta_{0}, \mathrm{~h}_{1}\right) \mathrm{y}_{\mathrm{n}}=4 \xi_{0}^{3} \mathrm{k} \frac{\mathrm{d}}{\mathrm{d} \xi_{0}} \mathrm{~h}_{0}^{(1)}\left(\xi_{0}\right) \mathrm{R}_{\mathrm{s} 0}\left(\theta_{0}\right), \\
\left.\sum_{\mathrm{n}=0}^{\infty} \mathrm{X}_{\mathrm{n}} \tilde{\mathrm{a}}_{\mathrm{ns}} \| \xi_{0}, \xi_{1}, \mathrm{~h}_{1}\right)+\sum_{\mathrm{n}=0}^{\infty} \mathrm{y}_{\mathrm{n}} \mathrm{b}_{\mathrm{ns}}\left(\xi_{1}\right)=-\mathrm{ik} \sum_{\mathrm{n}=0}^{\infty} \mathrm{B}_{0 \mathrm{n}}\left(\mathrm{h}_{1}\right) \mathrm{a}_{\mathrm{ns}}\left(\xi_{1}\right), \mathrm{s}=0,1,2, \ldots,
\end{array}\right\}
$$

where

$$
\tilde{\mathrm{b}}_{\mathrm{ns}}\left(\xi_{0}, \theta_{0}, \mathrm{~h}_{1}\right)=4 \mathrm{i} \xi_{0}^{3} \sum_{\mathrm{p}=0}^{\infty} \frac{\mathrm{d}}{\mathrm{d} \xi_{0}} \mathrm{j}_{\mathrm{p}}\left(\xi_{0}\right) \mathrm{R}_{\mathrm{sp}}\left(\theta_{0}\right) \mathrm{A}_{\mathrm{np}}\left(\mathrm{h}_{1}\right) /(2 \mathrm{p}+1)
$$

\section{Calculation of the far field}

On the basis of formula:

$$
\left.\begin{array}{l}
\mathrm{h}_{\mathrm{n}}^{(1)}\left(\mathrm{kr}_{1}\right) \mathrm{P}_{\mathrm{n}}\left(\cos \theta_{1}\right)=\sum_{\mathrm{p}=0}^{\infty} \tilde{\mathrm{A}}_{\mathrm{np}} \backslash \mathrm{h}_{1} \mid \mathrm{h}_{\mathrm{p}}^{(1)}(\mathrm{kr}) \mathrm{P}_{\mathrm{p}}(\cos \theta), \mathrm{r}>\mathrm{h}_{1}, \\
\underline{\tilde{A}}_{\mathrm{np}} \backslash \mathrm{h}_{1} \mid=\sum_{\sigma=|\mathrm{p}-\mathrm{n}|}^{\mathrm{p}+\mathrm{n}}(2 \sigma+1) \mathrm{i}^{\sigma+\mathrm{p}-\mathrm{n}} \mathrm{b}_{\mathrm{p}}^{(\mathrm{n} 0 \sigma 0)} \mathrm{j}_{\sigma}\left(\mathrm{kh}_{1}\right)
\end{array}\right\}
$$

we have representation of the function $\mathrm{p}_{2}^{(2)}\left(\mathrm{r}_{1}, \theta_{1}\right)$ in coordinate system with origin at the point $\mathrm{O}$

$$
\mathrm{p}_{2}^{(2)}(\mathrm{r}, \theta)=\mathrm{P} \sum_{\mathrm{n}=0}^{\infty} \mathrm{U}_{\mathrm{n}} \mathrm{h}_{\mathrm{n}}^{(1)}(\mathrm{kr}) \mathrm{P}_{\mathrm{n}}(\cos \theta), \mathrm{U}_{\mathrm{n}}\left(\mathrm{h}_{1}\right)=\sum_{\mathrm{p}=0}^{\infty} \tilde{\mathrm{A}}_{\mathrm{pn}} \| \mathrm{h}_{1} \mid \mathrm{y}_{\mathrm{p}}
$$

Using the asymptotic expression for the function $h_{n}^{(1)}(k r)[16]$ :

$$
\mathrm{h}_{\mathrm{n}}^{(1)}(\mathrm{kr}) \approx(-\mathrm{i})^{\mathrm{n}+1} \mathrm{e}^{\mathrm{ikr}} / \mathrm{kr}, \quad \mathrm{kr} \rightarrow \infty
$$

we obtain representation of pressure in the far field zone:

$$
\mathrm{p}_{2}(\mathrm{r}, \theta)=\mathrm{P} \frac{\mathrm{e}^{\mathrm{ikr}}}{\mathrm{kr}} \mathrm{G}(\theta)
$$

where 


$$
G(\theta)=\sum_{n=0}^{\infty}(-i)^{n+1}\left(X_{n} \frac{d}{d \xi_{0}} j_{n}\left(\xi_{0}\right)+f_{n}+\sum_{p=0}^{\infty} \tilde{A}_{p n}\left(h_{1}\right) y_{p}\right) P_{n}(\cos \theta)
$$

The function $\mathrm{G}(\theta)$ for some parameters of the problem is calculated using a computer algebra system Mathcad [21]. Spherical functions were calculated by means of built-in functions. Derivatives of spherical functions were calculated by means of the recurrent formulas [17]. The infinite system (36) was solved by the method of truncation [16]. The computational experiment showed that the truncation order for the considered parameters of the problem can be equal to 25 . It provides the solution of the system (36) with accuracy $10^{-4}$. Figure 2 shows plots of the function $G(\theta)$ for some values of the angle $\theta_{0}$ of thin unclosed spherical shell $\Gamma_{1}$. The parameters are equal to: $\mathrm{h}_{1}=1.0 \mathrm{~m}, \mathrm{a}=0.2 \mathrm{~m}, \mathrm{~b}=0.9 \mathrm{a}, \mathrm{k}=1.5$ $\mathrm{m}^{-1}$. Figure 3 shows plots of the function $\mathrm{G}(\theta)$ for some values of the wave number $\mathrm{k}$. The parameters are equal to: $\mathrm{h}_{1}=1.0 \mathrm{~m}, \mathrm{~d}=0.2 \mathrm{~m}, \mathrm{a}=0.2 \mathrm{~m}, \mathrm{~b}=0.9 \mathrm{a}, \theta_{0}$ $=90^{\circ}$. Figure 4 shows plots of the function $\mathrm{G}(\theta)$ for some values $\mathrm{b} / \mathrm{a}$ and parameters are equal to: $\mathrm{h}_{1}=0.7 \mathrm{~m}, \mathrm{~d}=0.2 \mathrm{~m}, \mathrm{a}=0.2 \mathrm{~m}, \mathrm{k}=4 \mathrm{~m}^{-1}, \theta_{0}=90^{0}$.

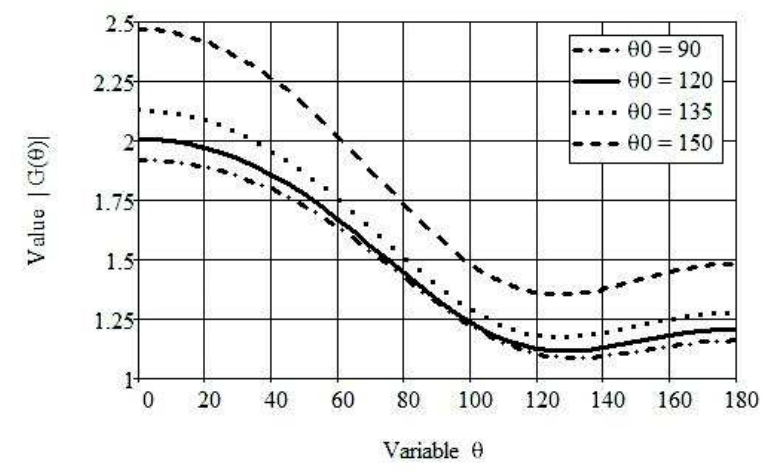

Fig. 2. Graph of function $G(\theta)$ for some values of the angle $\theta_{0}$

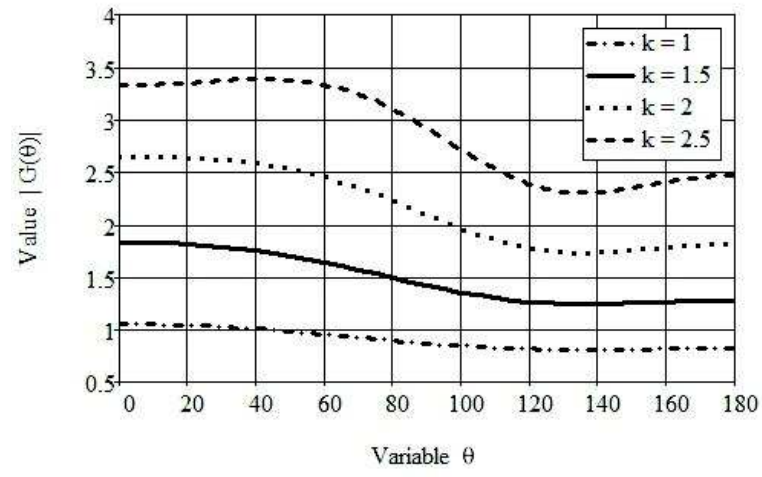

Fig. 3. Graph of function $G(\theta)$ for some values of the wave number $k$ 


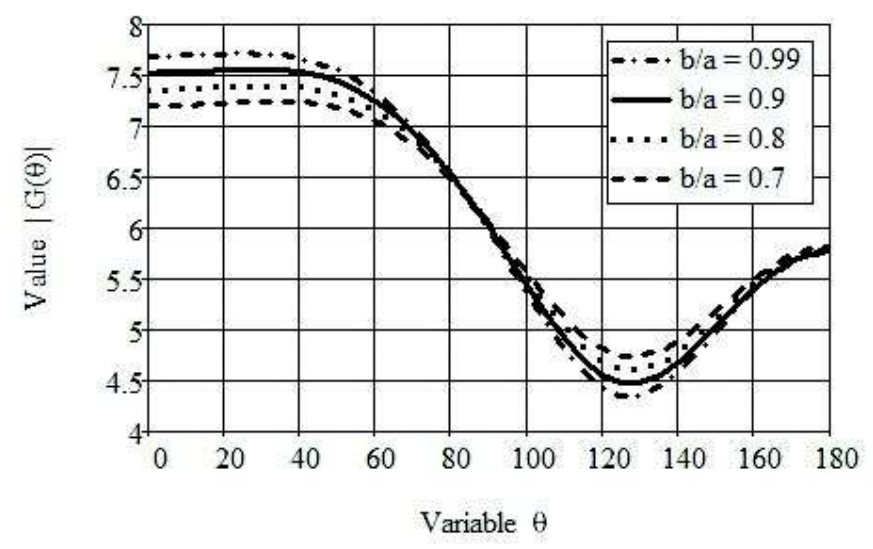

Fig. 4. Graphs of function $G(\theta)$ for some values $b / a$

\section{Conclusions}

The solution of the problem of the scattering of sound field by unclosed spherical shell and a soft prolate ellipsoid is reduced to solving dual equations in Legendre's polynomials using the addition theorem for spherical wave functions. The spherical radiator is considered as the source of the sound field located within the thin unclosed spherical shell. The equation of spheroidal boundary is considered in spherical coordinates. Following tasks were carried out:

- scattered pressure field is expressed in terms of spherical wave functions,

- dual equations are converted to the infinite system of linear algebraic equations of the second kind with the completely continuous operator,

- numerical results for various values of the parameters of the problem were computed.

The developed methodology and the software can be practically used in the manufacture of sound screens.

\section{Appendix}

The values of the integrals $\mathrm{I}_{\mathrm{ns}}^{(\alpha)}$.

Using recurrence relations for Legendre polynomials

$$
\left.\begin{array}{l}
x^{2} P_{n}(x)=\frac{n(n-1)}{(2 n-1)(2 n+1)} P_{n-2}(x)+\frac{2 n^{2}+2 n-1}{(2 n-1)(2 n+3)} P_{n}(x)+ \\
+\frac{(n-1)(n+2)}{(2 n+)(2 n+3)} P_{n+2}(x),
\end{array}\right\}
$$




$$
\left.\begin{array}{l}
x^{4} P_{n}(x)=\frac{n(n-1)(n-2)(n-3)}{(2 n+1)(2 n-1)(2 n-3)(2 n-5)} P_{n-4}(x)+ \\
+\frac{n(n-1)\left(4 n^{2}-4 n-14\right)}{(2 n-5)(2 n-1)(2 n+1)(2 n+3)} P_{n-2}(x)+\frac{3\left(2 n^{4}+4 n^{3}-2 n^{2}-8 n+3\right)}{(2 n-3)(2 n-1)(2 n+3)(2 n+5)} P_{n}(x)+ \\
+\frac{(n+1)(n+2)\left(4 n^{2}+12 n-6\right)}{(2 n-1)(2 n+1)(2 n+3)(2 n+7)} P_{n+2}(x)+\frac{(n+1)(n+2)(n+3)(2+4)}{(2 n+1)(2 n+3)(2 n+5)(2 n+7)} P_{n+4}(x)
\end{array}\right\}
$$

and the value of the integral

$$
\mathrm{I}_{\mathrm{sn}}^{(1)}=\int_{0}^{\pi} \mathrm{P}_{\mathrm{n}}(\cos \theta) \mathrm{P}_{\mathrm{s}}(\cos \theta) \sin \theta \mathrm{d} \theta=\left\{\begin{array}{l}
\frac{2}{2 \mathrm{n}+1}, \mathrm{~s}=\mathrm{n}, \\
0, \mathrm{~s} \neq \mathrm{n},
\end{array}\right.
$$

we obtain the following values of integrals

$$
I_{\mathrm{sn}}^{(3)}= \begin{cases}\frac{-2 n(n-1)}{(2 n-3)(2 n-1)(2 n+1)}, & s=n-2, \\ \frac{4\left(s^{2}+s-1\right)}{(2 s-1)(2 s+1)(2 s+3)}, & s=n, \\ \frac{-2(n+1)(n+2)}{(2 n+1)(2 n+3)(2 n+5)}, & s=n+2, \\ 0, s \neq n,\end{cases}
$$




$$
I_{\mathrm{sn}}^{(3)}= \begin{cases}\frac{2 n(n-3)(n-2)(n-1)}{(2 n-7)(2 n-5)(2 n-3)(2 n-1)(2 n+1)}, & s=n-4, \\ \frac{8 n(n-1)\left(-n^{2}+n+4\right)}{(2 n-5)(2 n-3)(2 n-1)(2 n+1)(2 n+3)}, & s=n-2 \\ \frac{4\left(3 n^{4}+6 n^{3}-8 n^{2}-14 n+12\right)}{(2 n-3)(2 n-1)(2 n+1)(2 n+3)(2 n+5)}, & s=n, \\ \frac{8(n+1)(n+2)\left(-n^{2}-3 n+2\right)}{(2 n-1)(2 n+1)(2 n+3)(2 n+5)(2 n+7)}, & s=n+2, \\ \frac{2(n+1)(n+2)(n+3)(n+4)}{(2 n+1)(2 n+3)(2 n+5)(2 n+7)(2 n+9)}, & s=n+4, \\ 0, s \neq n, & \end{cases}
$$

$$
I_{\mathrm{sn}}^{(7)}= \begin{cases}\frac{-2(n-5)(n-4)(n-3)(n-2)(n-1) n}{(2 n-11)(2 n-9)(2 n-7)(2 n-5)(2 n-3)(2 n-1)(2 n+1)}, & s=n-6, \\ \frac{-12 n(n-3)(n-2)(n-1)\left(-n^{2}+3 n+7\right)}{(2 n-9)(2 n-7)(2 n-5)(2 n-3)(2 n-1)(2 n+1)(2 n+3)}, & s=n-4, \\ \frac{-6 n(n-1)\left(5 n^{4}-10 n^{3}-59 n^{2}+64 n+180\right)}{(2 n-7)(2 n-5)(2 n-3)(2 n-1)(2 n+1)(2 n+3)(2 n+5)}, & s=n-2, \\ \frac{8\left(5 n^{6}+15 n^{5}-52 n^{4}-129 n^{3}+155 n^{2}+222 n-180\right)}{(2 n-5)(2 n-3)(2 n-1)(2 n+1)(2 n+3)(2 n+5)(2 n+7)}, & s=n, \\ \frac{-6(n+1)(n+2)\left(5 n^{4}+30 n^{3}+n^{2}-132 n+72\right)}{(2 n-3)(2 n-1)(2 n+1)(2 n+3)(2 n+5)(2 n+7)(2 n+9)} & \text { if } s=n+2 \\ \frac{12(n+1)(n+2)(n+3)(n+4)\left(n^{2}+5 n-3\right)}{(2 n-1)(2 n+1)(2 n+3)(2 n+5)(2 n+7)(2 n+9)(2 n+11)}, & s=n+4, \\ \frac{-2(n+1)(n+2)(n+3)(n+4)(n+5)(n+6)}{(2 n+1)(2 n+3)(2 n+5)(2 n+7)(2 n+9)(2 n+11)(2 n+13)}, & s=n+6, \\ 0, n \neq s . & \end{cases}
$$




\section{Acknowledgement}

The research leading to these results has supported by funding from the People Programme (Marie Curie International Research Staff Exchange) of the EU FP7/2007-2013/ under REA grant agreement $n^{\circ}$ PIRSES-GA-2013-610547.

\section{References}

[1] Kleshchev A.A., Sheiba L.S.: Scattering of a sound wave by ideal prolate spheroids, Acoustic J., 16 (1970) 264-268 (in Russian).

[2] Sidman R.D.: Scattering of acoustical waves by a prolate spherical obstacle, J. Acoust. Soc. America, 52 (1972) 879-883.

[3] Lauchle G.C.: Short-wavelength acoustic diffraction by prolate spheroids. J. Acoust. Soc. America, 58 (1975) 568-575.

[4] Germon A., Lauchle G.C.: Axisymmetric diffraction of spherical waves by a prolate spheroid, J. Acoust. Soc. America, 65 (1979) 1322-1327.

[5] Varadan V.K., Varadan V.V., Dragonette L.R., Flax L.: Computation of rigid body by prolate spheroids using the T-matrix approach, J. Acoust. Soc. America, 71 (1982), 22-25.

[6] Sammelmann G.S., Trivett D.H., Hackmann R.H.: High-frequency scattering from rigid prolate spheroids, J. Acoust. Soc. America, 83 (1988) 46-54.

[7] Barton J.P., Wolf N.L., Zhang H., Tarawneh C.: Near-field calculations for a rigid spheroid with an arbitrary incident acoustic field, J. Acoust. Soc. America, 103 (2003) 1266-1222.

[8] Burke J.E.: Scattering by penetrable spheroids, J. Acoust. Soc. America, 43 (1968) 871-875.

[9] Kotsis A.D., Roumeliotis J.A.: Acoustic scattering by a penetrable spheroid, Acoust. Phys., 54 (2008) 153-167.

[10] Kleshchev A.A., Rostovcev D.M.: Scattering of a sound by elastic and liquid ellipsoidal shells of revolution, Acoustic J., 32 (1986) 691-694 (in Russian).

[11] Kleshchev A.A. With reference to low frequency resonances of elastic spheroidal bodies, J. Techn. Ac., 2 (1995) 27-28.

[12] Bao X.L., Uberall H., Niemiec J.: Experimental study of sound scattering by elastic spheroids, J. Acoust. Soc. America, 102 (1997) 933-942.

[13] Tolokonnikov L. A., Lobanov A. V.: About scattering of plane sound wave by inhomogeneous elastic spheroid, Proc. Tula Stat. Univ. Natural Sci., 3 (2011) 119125 (in Russian).

[14] Tolokonnikov L. A.: Diffraction of plane sound wave on elastic spheroid with arbitrary located spherical vacuity, Proc. Tula State Univ. Natural Sci., 2 (2011) 169175 (in Russian).

[15] Grinchenko V.T., Vovk I.V., Matsipura V.T.: Fundamentals of acoustics, Naukova dumka, Kiev 2007 (in Russian).

[16] Ivanov E. A.: Diffraction of electromagnetic waves on two bodies, Springfield, Washington 1970. 
[17] Handbook of Mathematical Functions: with Formulas,Graphs and Mathematical Tables, Eds. by M. Abramowitz and I. A. Stegun, Dover, New York 1972.

[18] Erofeenko V.T.: Addition theorems, Nauka i Technika, Minsk 1989 (in Russian).

[19] Shushkevich G.Ch., Kiselyova N.N.: Penetration of sound field through multilayered spherical shell, Informatika, 3 (2013) 47-57 (in Russian).

[20] Rezunenko V.A. Diffraction of plane acoustic wave on sphere with circular aperture, Bulletin Kharkiv Nat. Univ., serie Mat., Appl. Mat. Mech., 850 (2009) 71-77 (in Russian).

[21] Shushkevich G.Ch., Shushkevich S.V.: Computer technology in mathematics. The system Mathcad 14: in 2 parts, Grevsova, Minsk 2012 (in Russian).

\title{
ROZPROSZENIE POLA AKUSTYCZNEGO ZA POMOCĄ CIENKIEJ NIEZAMKNIETEJ KULISTEJ POWLOKI ORAZ ELIPSOIDY
}

\begin{abstract}
Streszczenie
W niniejszym opracowaniu zaprezentowano wyniki rozwiązania osiowosymetrycznego problemu rozproszenia pola dźwiękowego przez niezamkniętą powłokę kulistą oraz lekko wydłużoną elipsoidę. Radiator kulisty znajdujący się w cienkiej niezamkniętej powłoce kulistej jest źródłem pola akustycznego. Równanie granicy kulistej podane jest we współrzędnych sferycznych. Rozproszone pole ciśnienia jest wyrażona w funkcji fal sferycznych. Stosując odpowiednie twierdzenia dodawania i przy założeniu zbyt małej mimośrodowości elipsy, rozwiązanie problemu wartości brzegowych jest ograniczone do rozwiązania podwójnych równań wielomianów Legendre'a, które przekształca się w nieskończony układ liniowych równań algebraicznych drugiego rodzaju z w pełni ciągłym operatorem. Wyniki obliczeń numerycznych są podane dla różnych wartości analizowanych parametrów.
\end{abstract}

Słowa kluczowe: pole akustyczne, kulista powłoka, elipsoida obrotowa, radiator kulisty

DOI: $10.7862 / \mathrm{rm} .2016 .14$

Otrzymano/received: 4.05.2016 r.

Zaakceptowano/accepted: $2.06 .2016 \mathrm{r}$. 